\title{
The effect of interleukin 17 and Toll-like receptor 2 on CD11b expression and apoptosis of neutrophils in zymosan- induced arthritis and paw oedema
}

\author{
VIKTORIYA MILANOVA, NINA IVANOVSKA, PETYA DIMITROVA
}

Department of Immunology, Institute of Microbiology, Sofia, Bulgaria

\begin{abstract}
We investigated the effect of interleukin (IL)-17 on Ly6G $G^{+}$cell apoptosis in zymosan-induced arthritis (ZIA) and oedema (ZIO). Zymosan injection at the ankle joint caused swelling and coincided with histological joint alterations and IL-17A expression in areas with cell infiltrates. Flow cytometry of blood demonstrated increased frequencies of $L y 6 G^{+} C D 11 b^{+}$cells and their decreased apoptosis in ZIA. Annexin $V^{+}$neutrophils had lower CD11b expression, unlike Annexin $V^{-}$cells. Cell survival for 12 hours was affected neither by IL-17 nor by zymosan alone, while both stimuli diminished Annexin $V^{+}$ cell frequencies and up-regulated CD11b on Annexin $V^{-}$cells. Interleukin 17 antagonised to the effects of zymosan in 24-hour cultures. The administration of IL-17 in ZIO increased paw thickness, enlarged the blood $L^{2} 6 G^{+}$pool, elevated CD1 1 b expression and decreased apoptosis. We suggest that altered neutrophil apoptosis in arthritis can be overcome by anti-IL-17 therapy combined with an inhibition of Toll-like receptor (TLR) 2 and CD11b signalling.
\end{abstract}

Key words: neutrophils, CD11b, interleukin 17, neutrophils, TLR2 ligand, paw oedema.

(Centr Eur J Immunol 2014; 39 (2): 131-141)

\section{Introduction}

Homeostasis of neutrophils in blood involves a coordinated process of granulopoiesis, mobilisation, constitutive apoptosis and clearance of apoptotic and senescent neutrophils. The homeostatic system is highly sensitive because neutrophils are terminally differentiated and shortlived cells. Apoptosis is a genetically controlled process of cell death, which results in removal of cell debris without causing inflammation. Apoptotic cells undergo functional, physiological and phenotype changes. The latter include the exposure of phosphatidylserine on cell membrane, the expression of cell death receptors and intracellular caspases [1]. Members of the tumour necrosis factor (TNF) receptor superfamily (TNFR1, TRAIL-R and CD95/FAS) activate a cascade that leads to the formation of a death-inducing signalling complex, activation of pro-caspase 8 and subsequent cleavage of pro-caspase 3 . The intrinsic pathway is triggered by various environmental factors and involves the members of the B-cell lymphoma $(\mathrm{Bcl})-2$ family and the expression and activation of pro-apoptotic mitochondrial proteins and caspases [1].

Blood neutrophils undergo constitutive apoptosis [2]. The kinetic of the process is dependent on a functional cross-talk between intrinsic pathway and signals initiated by death receptor activation [3]. The abnormalities in the pro- and anti-apoptotic mechanisms and/or the exposure to stress factors alter neutrophil apoptosis and are often related to disease pathology. In patients with rheumatoid arthritis (RA), an inflammatory disease affecting joints, cartilage and bone, neutrophils have delayed apoptosis in synovial fluid and blood because of elevated levels of interleukin (IL)-1, tumor necrosis factor $\alpha$ (TNF- $\alpha$ ), IL-8, granulocyte colony-stimulating factor (G-CSF) and granulocyte-macrophage colony-stimulating factor (GM-CSF) associated with the disease pathology $[4,5]$. It has been shown that GM-CSF is involved in arthritic and inflammatory progression and pain development [6]. This factor controls constitutive apoptosis via up-regulated expression of anti-apoptotic genes, genes related with inflammatory response, genes encoding major histocompatibility complex class II molecules, proteasome subunits and ubiquitin-modifying enzymes [7]. In addition, it regulates cell survival by interfering with modulators of apoptosis like myeloid leukaemia cell differentiation protein (Mcl-1) [8] and signalling pathways including phosphatidylinositide 3-kinase (PI3K) [9], and by preventing the formation of the

Correspondence: Assoc. Prof. Petya Dimitrova, Department of Immunology, Institute of Microbiology, 26 Georgi Bonchev Str., 1113 Sofia, Bulgaria, e-mail: petya_dimitrova@web.de 
death-inducing signalling complex required for FAS-induced apoptosis [10].

Interleukin 17 is a pro-inflammatory cytokine involved in RA pathology, which has recently been considered to be a potential therapeutic target for the disease [11]. It acts on chemokines, cytokines and inflammatory mediator genes, antimicrobial peptides and transcription factors [12]. The biological effects of IL-17 depend on the formation of IL-17RA/IL-17RC heterodimer receptor complex [12]. Cells that are able to express both receptors like fibroblasts and chondrocytes are sensitive to the cytokine and upon strong stimulation can undergo apoptosis $[13,14]$. In respect to neutrophils, IL-17 regulates granulopoiesis, cell mobilisation and migration to the inflammatory sites. Previously we found that in zymosan-induced arthritis, neutrophils were positive for IL-17 and interferon (IFN)- $\gamma$ in synovial fluid and produced IL-17 spontaneously in blood [15]. Indeed, neutrophils from healthy mice synthesised IL-17 in response to exogenous IL-17 [15, 16]. Herein, we have extended our research and we have investigated the effect of IL-17 on neutrophil apoptosis.

\section{Material and methods}

\section{Mice}

$\mathrm{BALB} / \mathrm{c}$ mice were purchased from Charles River Laboratories (USA), kept under standard conditions of a 12-12 hour light-dark cycle and fed with a laboratory diet and water ad libitum. All experiments were approved by the Animal Care Committee at the Institute of Microbiology, Sofia in accordance with the National and European Guidelines.

\section{Zymosan-induced arthritis}

Female mice ( 6 weeks old) were anesthetised by intra-peritoneal injection (i.p.) of sodium pentobarbital (50 mg/kg; Sigma-Aldrich, Munich, Germany) supplemented with analgesic buprenorphine hydrochloride $(0.1 \mathrm{mg} / \mathrm{kg}$; Sigma-Aldrich). Mice were injected at the tibio-tarsal joint (ankle joint) with $10 \mu \mathrm{l}$ of zymosan suspension $(20 \mathrm{mg}$ / $\mathrm{ml}$ ) or $10 \mu \mathrm{l}$ of endotoxin free phosphate-buffered saline (PBS; BioWittaker ${ }^{\circledR}$, Lonza, Verviers, Belgium). The ankle thickness was measured in millimetres at different time points by using a dial-type vernier calliper (Fisher Scientific, Schwerte, Germany). The results were expressed as the difference $(\Delta)$ in $\mathrm{mm}$ of the measurements before (baseline) and after zymosan injection.

\section{Histology}

At day 7 and 30 post-zymosan injection ankle joints were dissected, fixed in $10 \%$ paraformaldehyde/PBS, decalcified in 5\% nitric acid for 1 week, dehydrated, embedded in paraffin, cut and stained with haematoxylin and eosin (H\&E). Histological evaluation of the sections was applied by two independent observers using light microscopy (Lei- ca Microsystems, Wetzlar, Germany) using a $1 \times 10$ and a $1 \times 40$ lens. Photos were taken with a DS-Ri1 Nikon camera (Nikon Instruments Europe, Amstelveen, Netherlands).

\section{Immunohistochemistry for interleukin 17A expression in the joints}

Ankle joint sections were deparaffinised and rehydrated, and antigen retrieval with $10 \mathrm{mM}$ sodium citrate $/ 0.05 \%$ Tween 20 (pH 6.0) was performed for 20 minutes at $95^{\circ} \mathrm{C}$ to unmask the antigenic sites in tissue specimens. Endogenous peroxidase was blocked with $3 \% \mathrm{H}_{2} \mathrm{O}_{2} / 60 \%$ methanol for 10 minutes followed by blocking of unspecific binding for 30 minutes with buffer containing $1 \%$ bovine serum albumin (BSA), $0.1 \%$ gelatine, $0.5 \%$ Triton X-100 (all from Sigma-Aldrich) and 0.01 M PBS (pH 7.2). The sections were washed three times with PBS/0.5\% Tween-20 and antibody (Ab) against IL-17A (clone TC11-18H10; Abcam, Cambridge, UK; $10 \mu \mathrm{g} / \mathrm{ml}$ ) and isotype rat control (Biolegend, London, UK; $10 \mu \mathrm{g} / \mathrm{ml}$ ) were added for one hour. Then biotinylated anti-rat IgG antibody (Thermo Fisher Pierce Scientific, Rockford, IL, USA; $1 \mu \mathrm{g} / \mathrm{ml}$ ) was added for 20 minutes followed by incubation for 30 minutes with HRP-conjugated avidin (Ultra-sensitive staining kit; Thermo Fisher Pierce Scientific). DAB substrate kit was used to detect the specific binding (Thermo Scientific, Rockford, IL, USA). Sections were counterstained with Gill's haematoxylin for 15 seconds and examined by a light microscope with a $1 \times 100$ lens.

\section{Zymosan-induced paw oedema}

Female BALB/c mice received a sub-plantar injection of $30 \mu \mathrm{l}$ zymosan suspension (20 mg/ml; Sigma-Aldrich) at the hind paw. The contralateral hind paw was injected with $30 \mu \mathrm{l}$ of PBS and was used as a control. Paw thickness at the medial-lateral direction was measured in mm $0,0.5$, 1, 2 and 4 hours post-zymosan injection using a calliper (Fisher Scientific). The results were expressed as a difference $(\Delta)$ between baseline measurements before and after zymosan/PBS injection in the paw.

\section{Interleukin 17 administration in zymosan- induced paw oedema}

Female BALB/c mice were injected intra-peritoneally (i.p.) with mouse recombinant IL-17 (PeproTech EC, London, UK; $0.5 \mu \mathrm{g} / 200 \mu \mathrm{l} / \mathrm{mice}$ ) or endotoxin free PBS (Bio$\mathrm{Wittaker}^{\circledR}$, Lonza; $200 \mu \mathrm{l}$ ) one hour before the induction of paw oedema. Mice were sacrificed after four hours and blood was collected and used for quantification of GMCSF or flow cytometry analysis of cell apoptosis.

\section{Blood collection}

At day 7 of zymosan-induced arthritis or 4 hours after the induction of paw oedema, mice were anesthetised with sodium pentobarbital. Blood was drawn from the retro-or- 
bital sinus by Pasteur pipette, collected in tubes with heparin (Sigma-Aldrich, $5 \mathrm{U} / \mathrm{ml}$ ) and used for serum preparation or flow cytometry analysis.

\section{Measurement of GM-CSF in serum}

Serum was obtained after centrifugation at $1000 \times \mathrm{g}$ for 15 minutes at $4^{\circ} \mathrm{C}$. Granulocyte-macrophage colony-stimulating factor was quantified in serum by commercial enzyme-linked immunosorbent assay (ELISA kit; Biolegend; detection limit $<8 \mathrm{pg} / \mathrm{ml}$ ). The samples were assayed in triplicate. The concentration of GM-CSF was calculated from a standard curve of the recombinant mouse protein using Gen5 Data Analysis Software (BioTek Instruments, Bad Friedrichshall, Germany).

\section{Purification of blood neutrophils and in vitro culture}

Neutrophils were purified from heparinised blood after centrifugation at $700 \times \mathrm{g}$ at room temperature in density gradients of 55\%/65\%/75\% Percoll/ $0.9 \% \mathrm{NaCl}$ (GE Healthcare Life Sciences, Freiburg, Germany) [17]. Neutrophils were collected from the $65 \% / 75 \%$ layer interface, washed and counted. The population consisted of $80-85 \%$ positive cells for Ly6G and more than $90 \%$ viable cells. For in vitro culture neutrophils were resuspended at a concentration of $1 \times 10^{6} / \mathrm{ml}$ in sterile complete RPMI-1640 medium (Biowhittaker ${ }^{\circledR}$; Lonza) containing $10 \%$ foetal calf serum (FCS), $2 \mathrm{mM}$ L-glutamine, $100 \mathrm{U} / \mathrm{ml}$ penicillin, 100 $\mu \mathrm{g} / \mathrm{ml}$ streptomycin (all from Sigma-Aldrich) without or with GM-CSF (PeproTech EC; mouse recombinant; 50 ng/ $\mathrm{ml}$ ). The cells were stimulated with zymosan (Sigma-Aldrich; $20 \mu \mathrm{g} / \mathrm{ml}$ ) in the absence or presence of IL-17 (Abcam; mouse recombinant, $40 \mathrm{ng} / \mathrm{ml}$ ) and cultured at $37^{\circ} \mathrm{C}$, $5 \% \mathrm{CO}_{2}$ for different time points (from 6 to 24 hours).

\section{Flow cytometry}

Whole blood collected in heparin-tubes $(200 \mu \mathrm{l})$ was pre-incubated with Ab against mouse CD16/CD32 (eBiosciences, Vienna, Austria; $1 \mu \mathrm{g} / \mathrm{ml}$ ) for 10 minutes at room temperature to block unspecific binding. Abs against Ly6G (clone 1A8; Biolegend) and CD11b (clone M1-70; Biolegend) were added in appropriate concentrations and incubated for 20 minutes. Blood samples were then immediately subjected to flow cytometry analysis. In other experiments purified neutrophils were resuspended at $1 \times 10^{5} / \mathrm{ml}$ in $2 \%$ FCS/PBS containing $1 \mathrm{mM}$ ethylenediaminetetraacetic acid (EDTA) and stained with Abs against Ly6G and $\mathrm{CD} 11 \mathrm{~b}$ for 15 minutes, washed and analysed with a flow cytometer (BD LSR II) using BD FACSDiva v6.1.2 software (Becton Dickinson GmbH, San Jose, CA, USA).

\section{Analysis of cell apoptosis}

Whole blood collected in heparin-tubes $(100 \mu \mathrm{l})$ from mice with ZIA or ZIO was immediately incubated with
Abs against Ly6G, CD11b (both from Biolegend) and Annexin V (Abcam) for 10 minutes and then analysed by flow cytometry. In some experiments freshly isolated neutrophils were incubated as described above in the presence or the absence of IL-17 and zymosan. Neutrophil apoptosis was determined after 0, 6, 12 and 24 hours by Annexin V Apoptosis Detection Kit (Abcam). Briefly, cells $\left(1 \times 10^{6}\right.$ cells/ml in binding buffer) were incubated with Annexin V (FITC labelled; $5 \mu \mathrm{l}$ ) and propidium iodide (PI; $5 \mu \mathrm{l}$ ) for 5 minutes and subjected immediately to flow cytometry analysis.

\section{Statistical analysis}

Statistical analysis was accomplished by InStat 3.0 and GraphicPad Prism 5.0 software (GraphPad Software, La Jolla, CA, USA). Data were expressed as mean \pm SEM. One-way analysis of variance (ANOVA) was performed to compare the ankle and paw thickness between groups and to calculate the statistical significance of the differences. For other data, the differences in the mean values between groups were analysed with the two-tailed Student's $t$ test. Differences were considered significant when $p<0.05$.

\section{Results}

\section{Arthritis induced by injection of zymosan at the tibiotarsal joint of BALB/c mice}

The injection of zymosan at the ankle joint induced swelling (Fig. 1A). Ankle thickness increased until days 5 and 7 in the ZIA group but not in control mice indicating early inflammation triggered by TLR2 ligand. Swelling in ZIA mice decreased between days 7 and 13 and was then elicited between days 22 and 30 as a result of chronic inflammation (Fig. 1A). Histologic analyses of sections from the tibiotalar joint showed peri-articular inflammation of the soft tissues, focal and synovial hyperplasia at day 7 of ZIA (Fig. 1B). We observed synovial hyperplasia, loss of chondrocytes (data not shown) and ankylosis of the joints with spanning of the talus bone at day 30 (Fig. 1B). Our data showed that the disease visual score estimated by swelling coincided with the histological changes in ZIA joints (Fig. 1A, B).

We then evaluated the expression of IL-17A in the joints by immunohistochemistry. We localised IL-17A ${ }^{+}$ chondrocytes in the cartilage of normal and ZIA mice at day 7. Most of the chondrocytes were hypertrophic in the ZIA group, unlike in the PBS controls (data not shown). Interleukin 17A was undetectable in cartilage at day 30, probably due to chondrocyte loss (Fig. 1C). At day 7, IL-17A was associated with hypertrophic bone and cell infiltrates in synovial membrane (Fig. 1D). IL-17A ${ }^{+}$cells in the infiltrates can be primed for IL-17 production and expression in arthritic synovium as well as in circulation, as we have previously shown $[15,16]$. 

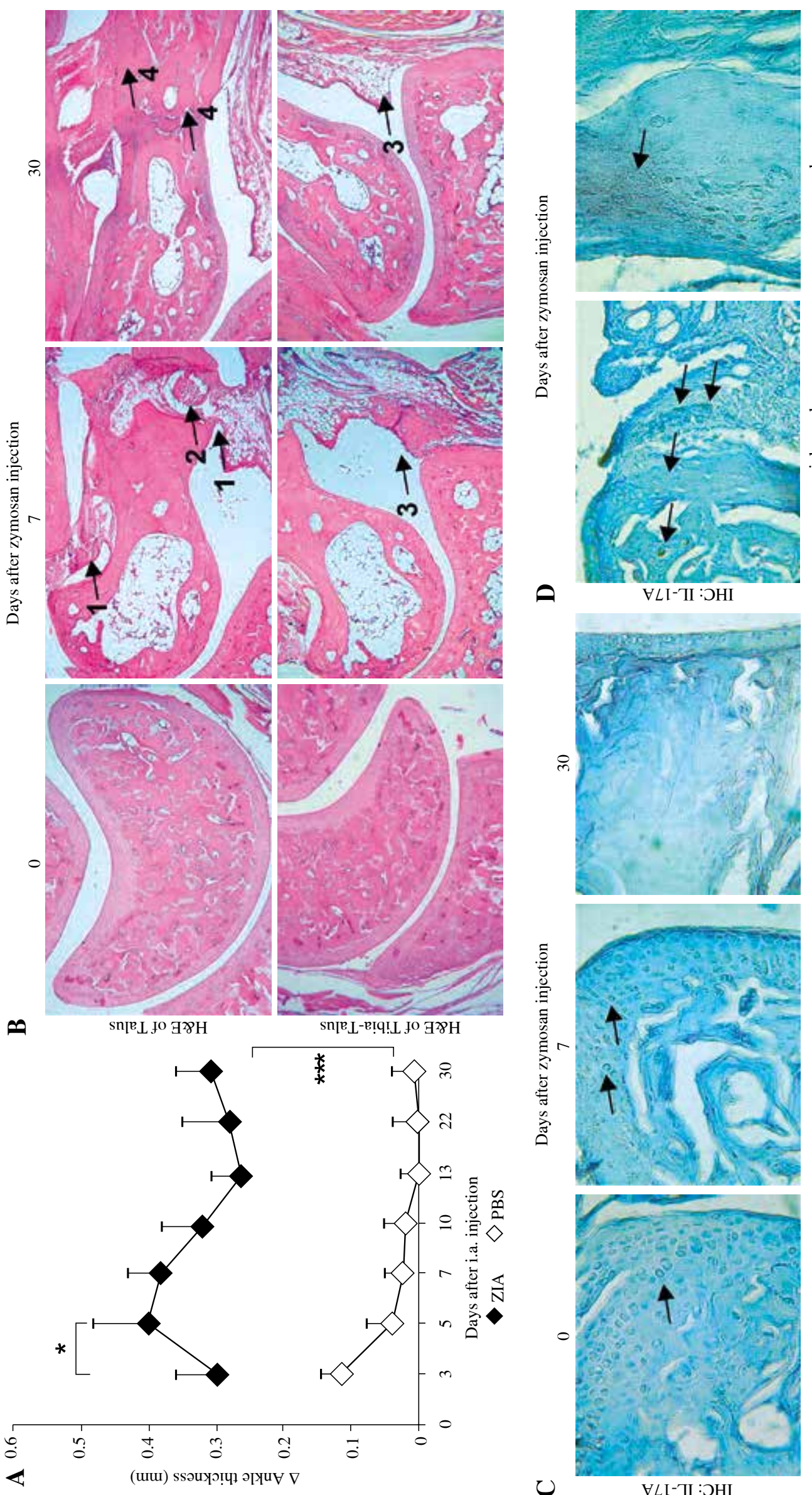

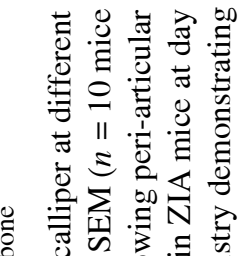

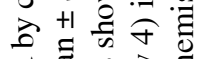
马 छ

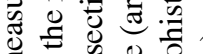

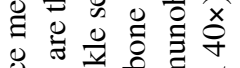

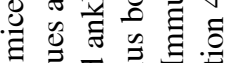

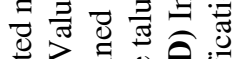

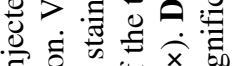

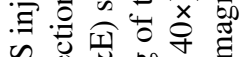

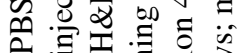

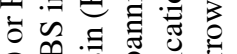
ये के की है 武要要志

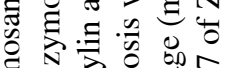

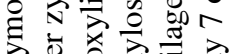

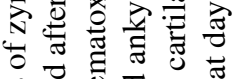

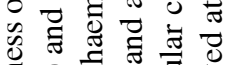

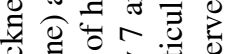
可完氖

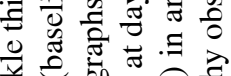

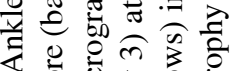
४ ه

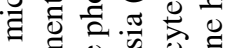
○ิ

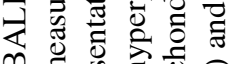

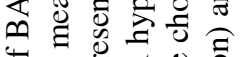

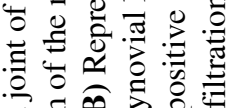

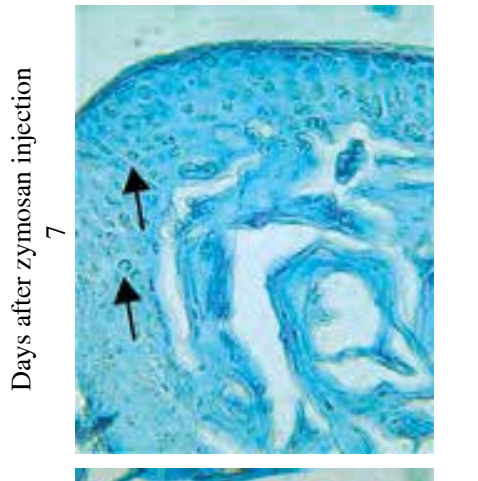

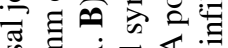

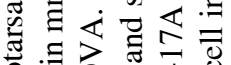

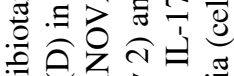
प

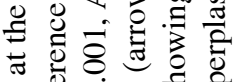

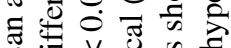

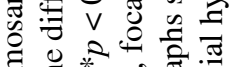

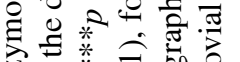

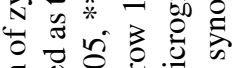

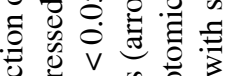

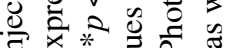

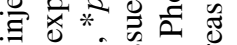

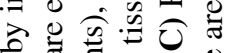

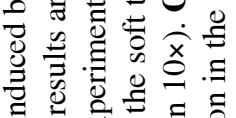
$\Xi$ 远

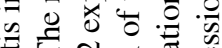

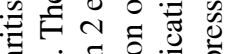

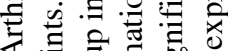

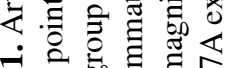

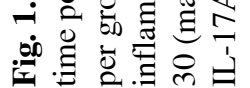




\section{Apoptosis of $\mathrm{Ly}^{6} \mathrm{G}^{+}$neutrophils in blood of mice with zymosan-induced arthritis}

Flow cytometry analysis of whole blood demonstrated increased frequencies of $\mathrm{Ly}_{6} \mathrm{G}^{+} \mathrm{CD} 11 \mathrm{~b}^{+}$cells from the ZIA group in comparison to the PBS group (Fig. 2A). $\mathrm{Ly}_{6 \mathrm{G}}{ }^{+}$cells expressing the early apoptotic marker Annexin $\mathrm{V}$ were fewer in the blood of ZIA mice than in controls (Fig. 2B). Annexin $\mathrm{V}^{+}$neutrophils showed lower CD11b expression, unlike Annexin $\mathrm{V}^{-}$cells (Fig. 2C). Decreased neutrophil apoptosis in ZIA mice was related to elicited production of GM-CSF in serum (Fig. 2D).

\section{Interleukin 17 and zymosan delay apoptosis and increase the CD11b expression on Annexin $\mathrm{V}^{-}$ neutrophils in vitro}

Subsequently, we evaluated the effect of IL-17 on apoptosis of blood neutrophils purified from PBS or ZIA mice. The survival of neutrophils from the PBS group decreased during culturing (Fig. 3A), while Ly6G ${ }^{+} \mathrm{CD} 11 \mathrm{~b}^{+}$ cells from ZIA mice were more resistant to apoptosis (Fig. 3B). Cell death was affected neither by IL-17 nor by zymosan alone for the period of 12 hours. Nevertheless, the presence of both stimuli diminished the frequencies of early apoptotic Annexin $\mathrm{V}^{+}$cells in PBS and ZIA mice (Fig. $3 \mathrm{~A}, \mathrm{~B})$. The cells that underwent apoptosis down-regulated CD11b in 6 hour cell cultures (data not shown), while both stimuli significantly increased the expression of CD11b on Annexin $\mathrm{V}^{-}$cells of the PBS and ZIA groups (Fig. 3C).

\section{Interleukin 17 antagonises the effects of zymosan on neutrophil apoptosis in the presence of granulocyte-macrophage colony-stimulating factor}

In the late period, 24-hour culture, neutrophil survival was sustained by GM-CSF (Fig. 4A, B) that mimicked arthritic conditions with elevated GM-CSF amounts in serum (Fig. 2D). Despite the presence of the survival factor, the frequencies of Annexin $\mathrm{V}^{+}$neutrophils from the PBS group were high and around 50\% (Fig. 4A). The number of Annexin $\mathrm{V}^{+}$cells decreased upon stimulation by TLR2 ligand but not by IL-17 (Fig. 4A). The frequencies of Annexin $\mathrm{V}^{+} \mathrm{Ly} 6 \mathrm{G}^{+} \mathrm{CD} 11 \mathrm{~b}^{+}$cells from ZIA mice were fewer in comparison to those from the PBS group. Zymosan accelerated the early apoptosis of ZIA neutrophils while IL-17 diminished the pro-apoptotic effect of the TLR2 ligand (Fig. 4B). Necrotic single $\mathrm{PI}^{+}$cells were detected in ZIA cultures but less in control ones (about 2\%; Fig. 4A, B).

\section{Administration of interleukin 17 in zymosan-induced oedema}

We then performed an experiment in which BALB/c mice were pre-treated with IL-17 and then injected with zymosan into the hind paw (Fig. 5A). Paw thickness was measured at indicated time points. The small differences between PBS-injected paws and the baseline at the beginning disappeared after four hours, indicating a lack of inflammation in the PBS group. TLR2 ligand induced paw oedema with maximal swelling 30 minutes post-injection. The pre-treatment with IL-17 significantly increased paw thickness in the zymosan-injected mice (Fig. 5B). The oedema remained for four hours in this group, in contrast to PBS mice (Fig. 5B).

Paw swelling in zymosan-injected mice was accompanied by enhanced frequencies of $\mathrm{Ly} 6 \mathrm{G}^{+}$cells in whole blood (Fig. 5C) and elevated expression of CD11b (Fig. 5D) on neutrophils, in comparison to PBS mice. Interleukin 17 pre-treatment expanded $\mathrm{Ly}_{6 \mathrm{G}^{+}}$cells in blood and amplified CD11b expression on neutrophils from ZIO mice, showing accelerated mobilisation and activation of mature neutrophils. In addition IL-17 enlarged the $\mathrm{Ly}_{6} \mathrm{G}^{+}$cell pool in $\mathrm{ZIO}$ mice through decreasing apoptosis (decreasing frequencies of Annexin $\mathrm{V}^{+}$neutrophils) (Fig. 5E). We were unable to find a difference in the serum GM-CSF amounts between IL-17-treated or untreated ZIO mice, suggesting that the effect of IL-17 on apoptosis was uncoupled to GM-CSF-delivered signals (Fig. 5F).

\section{Discussion}

Neutrophils are important players in the initiation of immune responses during inflammation and in inflammatory diseases like arthritis. In these conditions various pro-inflammatory signals can contribute to altered neutrophil functions, numbers and apoptosis.

The injection of TLR2 ligand in the ankle induced swelling with a peak between days 5 and 7, corresponding to the histological alterations of the joints. We detected IL-17A $\mathrm{A}^{+}$chondrocytes in cartilage of PBS mice as well as in ZIA mice at day 7. It has been shown that IL-17A is localised on hypertrophic chondrocytes expressing IL-17R, allowing a regulation of the IL-17 secretion in an autocrine manner [14]. We observed a loss of chondrocytes and negative staining for IL-17A in cartilage at late stage of arthritis (day 30). The link between IL-17 signalling and apoptosis of chondrocytes has been found in IL-17R-deficient mice where the lack of IL-17R prevents chondrocyte death [18].

Interleukin 17 is secreted at the initial step of inflammatory response and precedes joint alterations in RA patients [19]. Beside chondrocytes, various cell types can contribute to IL-17 production in synovial fluid. We observed IL-17A expression at areas with synovial hyperplasia. Similarly in arthritis induced by injections of gram-positive bacterial SCW fragments, IL-17 mRNA increases in the hyperplastic synovial tissue [18]. In ZIA mice we detected IL-17A ${ }^{+}$ staining at the synovial membrane regions with cell infiltrates. In RA patients IL-17A is co-localised to CD15+ neutrophils in the inflamed tissues [20]. Neutrophils in cell 


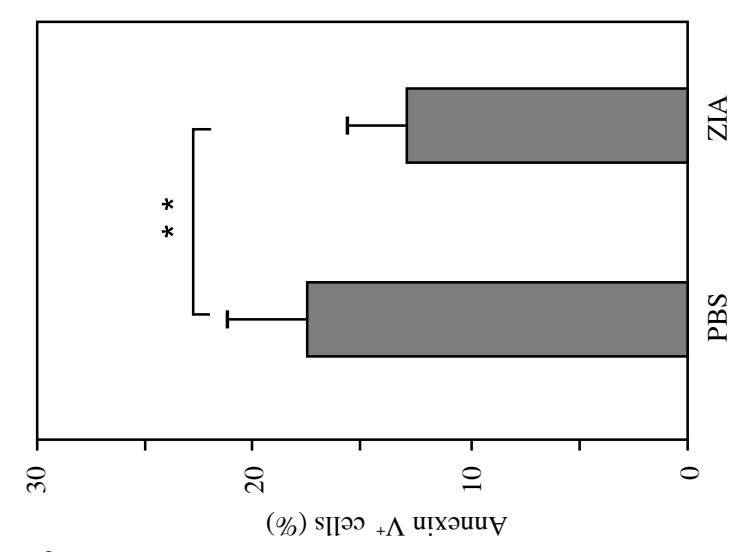

คి

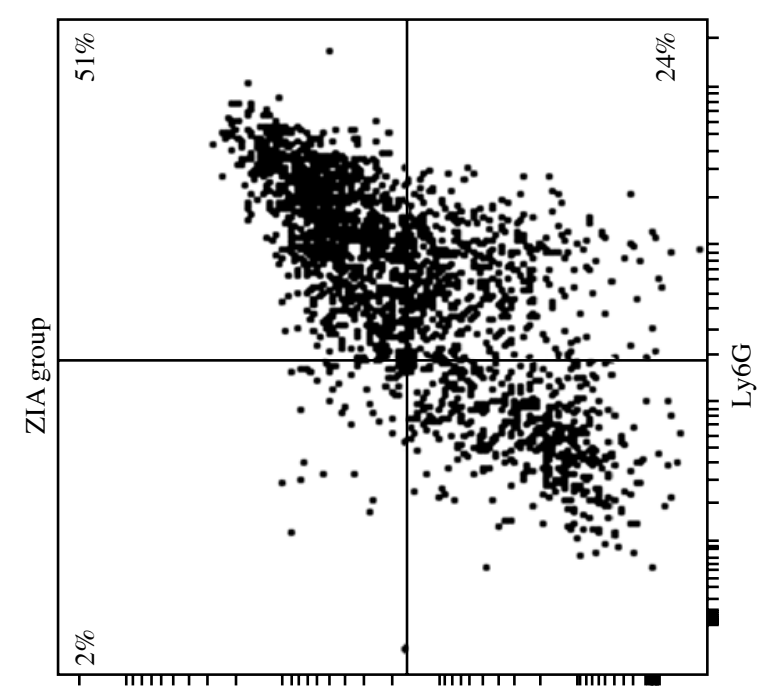

qIICO
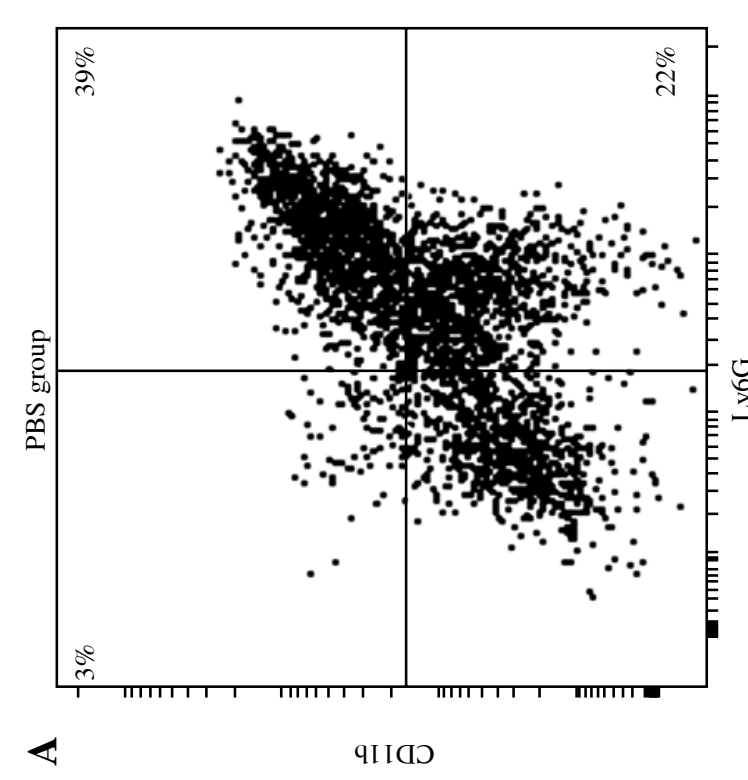

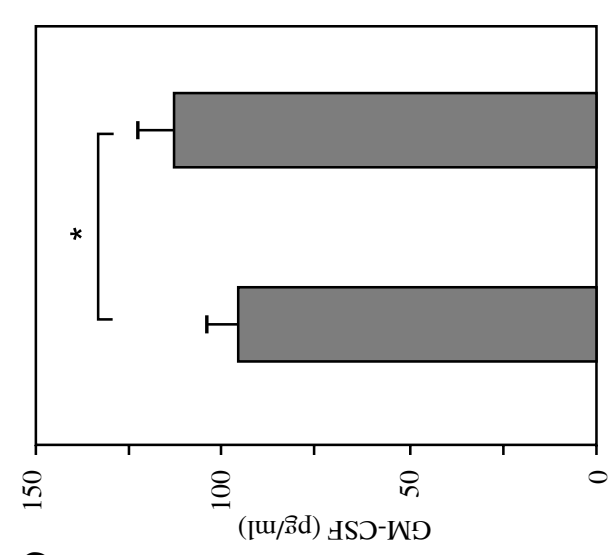

D

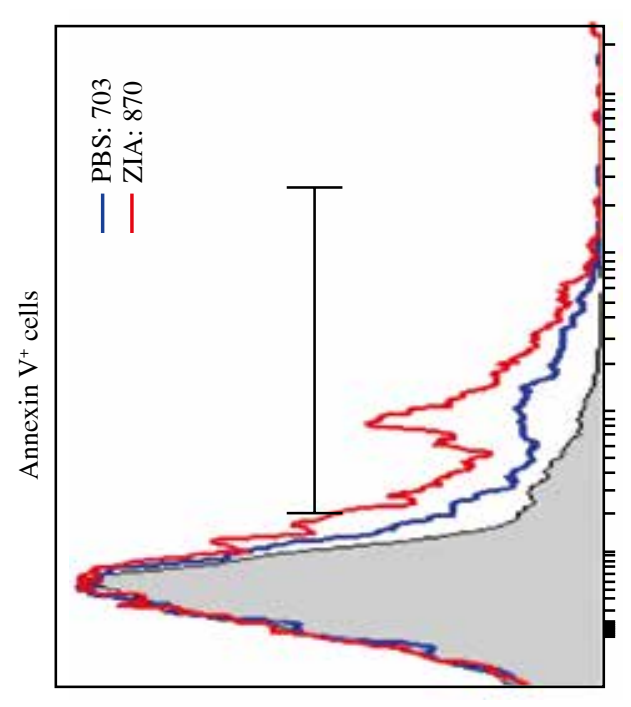

IHW

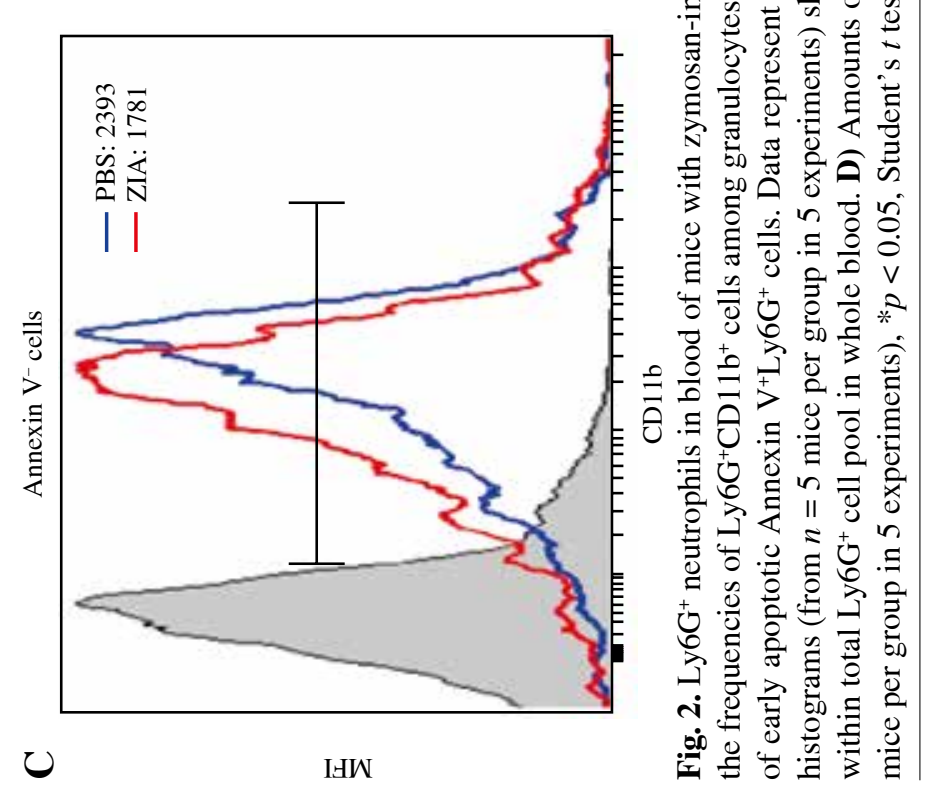


A

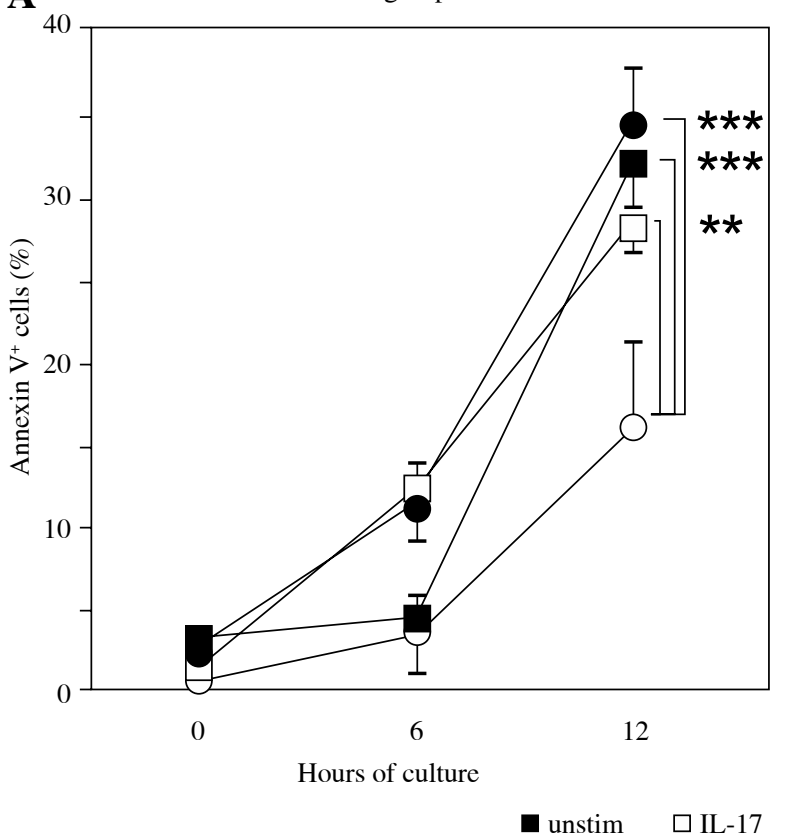

B

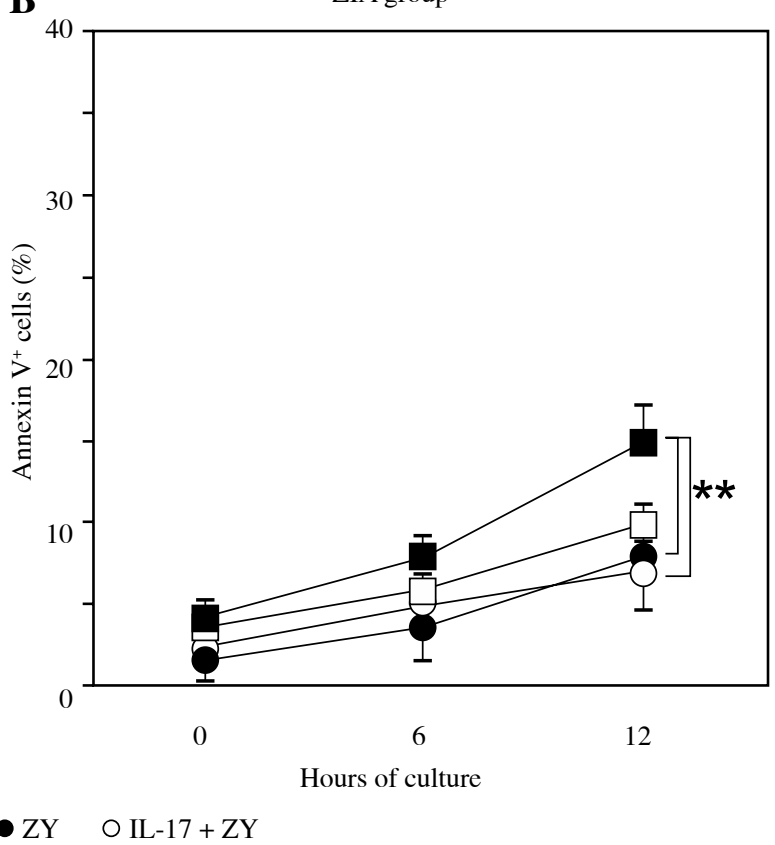

C

Annexin $\mathrm{V}^{-}$cells

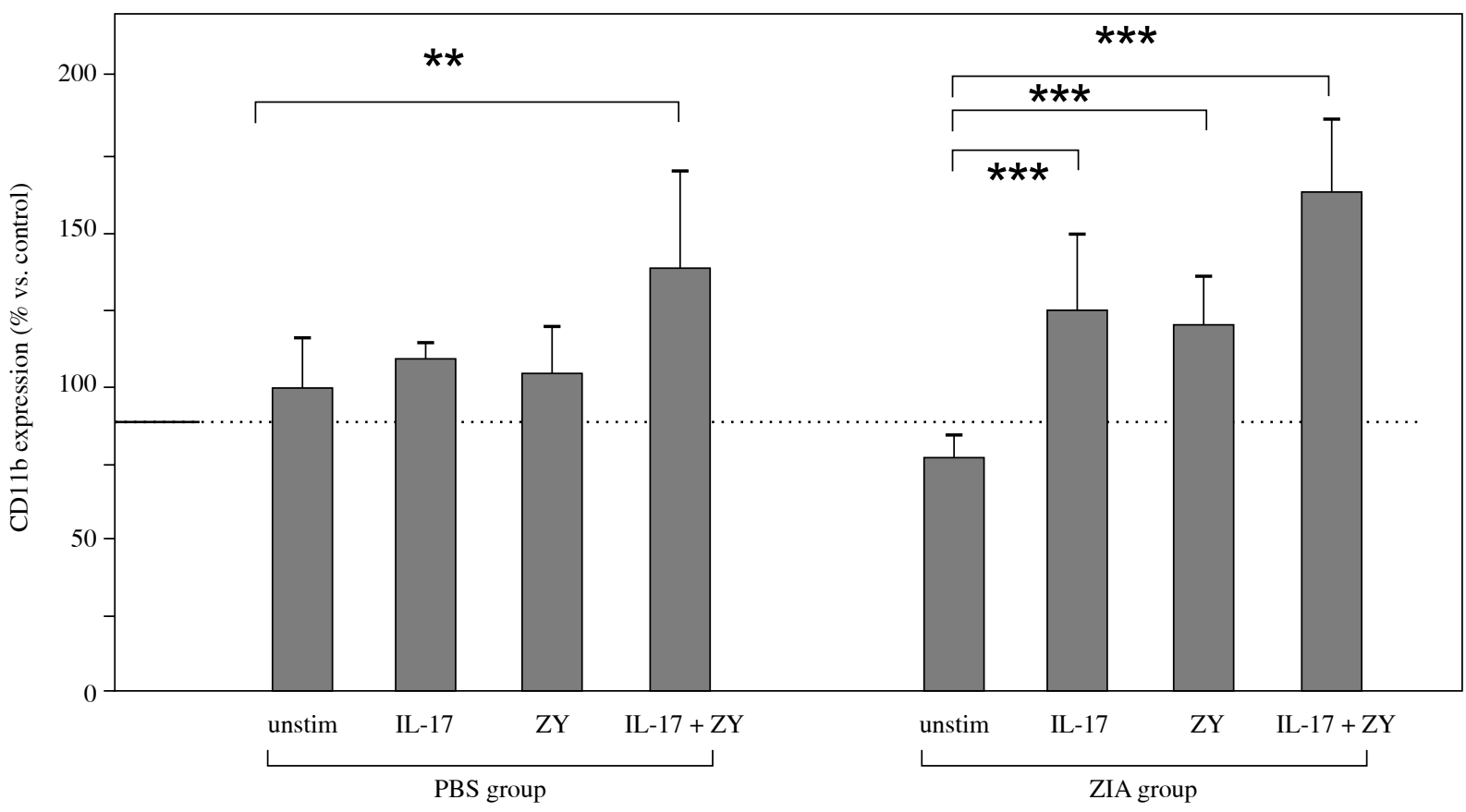

Fig. 3. Effect of IL-17 and TLR2 stimulation on the dynamic of Ly6G+ cell apoptosis. Apoptosis of purified blood Ly $6 \mathrm{G}^{+} \mathrm{CD} 11 \mathrm{~b}^{+}$cells from A) PBS group and B) ZIA group during culture period of 12 hours, $37^{\circ} \mathrm{C}, 5 \% \mathrm{CO}_{2}$ measured by commercial Annexin V kit. Neutrophils were stimulated with zymosan $(20 \mu \mathrm{g} / \mathrm{ml})$ in the presence or the absence of IL-17 $(40 \mathrm{ng} / \mathrm{ml})$. Apoptosis was evaluated at each time point in 6 samples per group and the values represent the mean \pm SEM of Annexin $\mathrm{V}^{+}$neutrophils. ${ }^{* *} p<0.01,{ }^{* * *} p<0.001$, Student's $t$ test. C) CD11b expression on Annexin $\mathrm{V}^{-}$neutrophils in cultures from the ZIA and PBS groups. Neutrophils from 6-hour cultures were collected, washed and stained with Abs against Ly6G and CD11b and then labelled with Annexin V. During flow cytometry analysis, Annexin $\mathrm{V}^{+}$and Annexin $\mathrm{V}^{-}$cells were gated within the $\mathrm{Ly} 6 \mathrm{G}^{+}$population. Mean fluorescent intensity (MFI) of CD11b on Annexin $\mathrm{V}^{-}$neutrophils was recorded. In order to compare different experiments, the values of MFI were normalised to the control of unstimulated neutrophils isolated from PBS group and expressed as a \% versus the control (dashed line). The values represent the mean $\pm \operatorname{SEM}(n=5$ animals per group in 3 experiments), $* * p<0.01, * * * p<0.001$, Student's $t$-test 
A

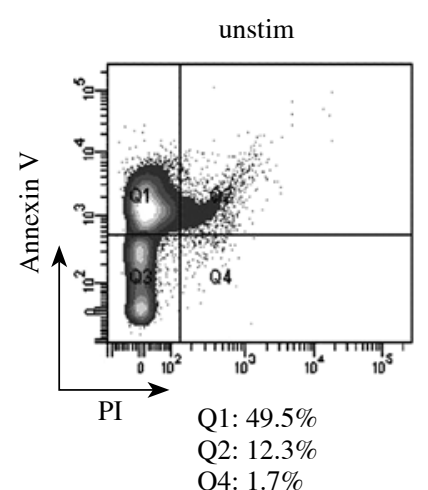

B
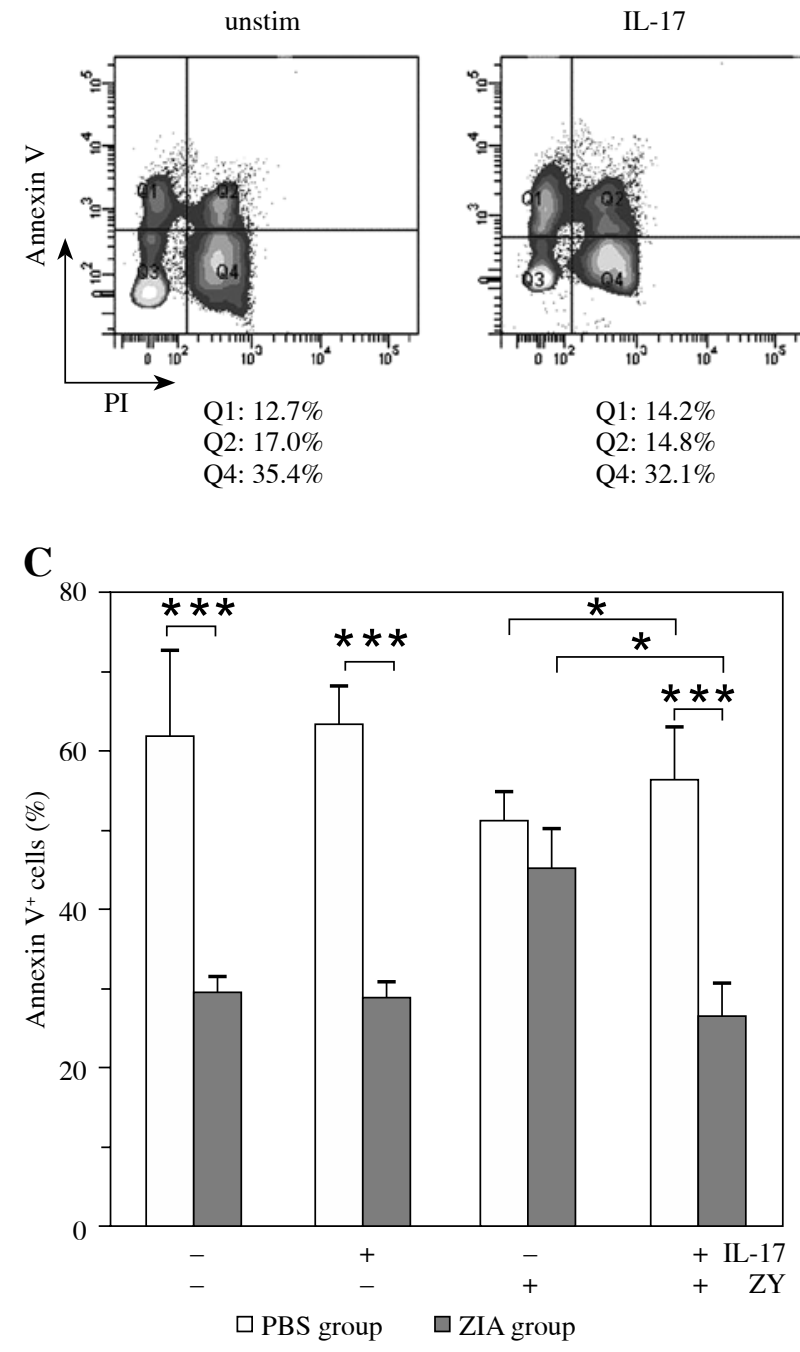

infiltrates can be primed for IL-17 synthesis already in circulation. Previously we found that blood neutrophils from mice with ZIA produced IL-17 spontaneously $[15,16]$. Migrating $\mathrm{Ly} 6 \mathrm{G}^{+} \mathrm{IL}-17^{+}$cells can become the initial source of IL-17 in synovial fluid, delivering signals for IL-17R-de-
PBS group
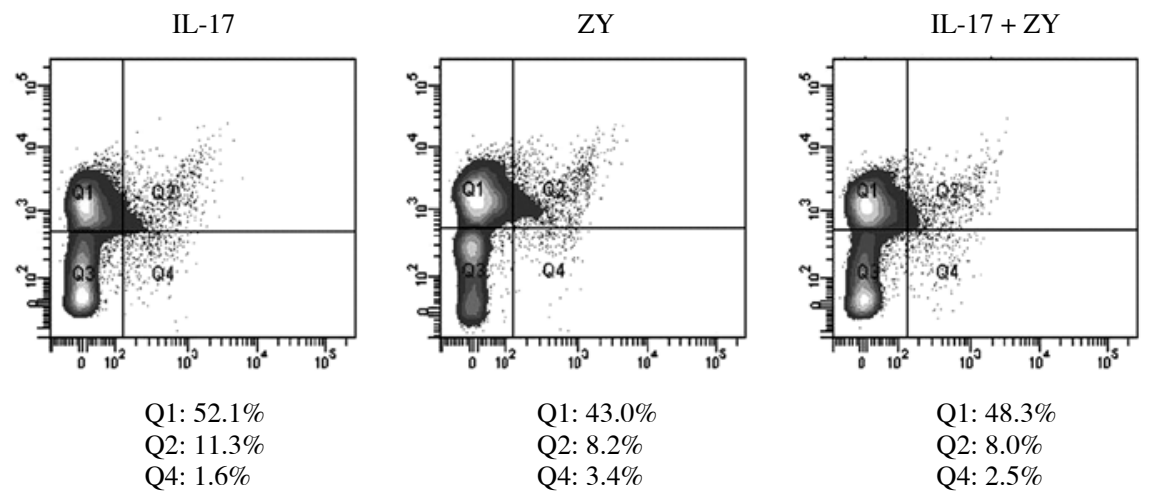

ZIA group
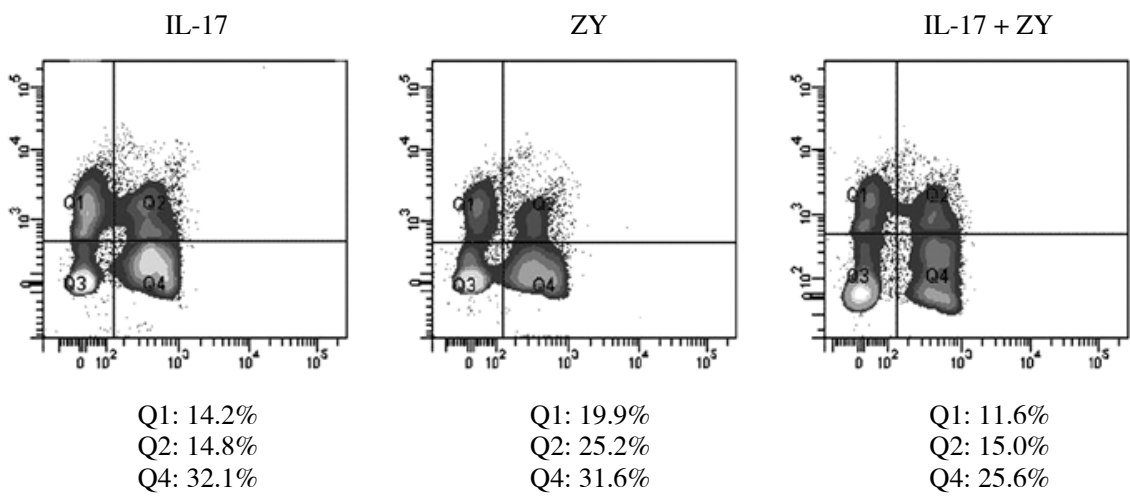

Fig. 4. Apoptosis of blood Ly $6 \mathrm{G}^{+} \mathrm{CD} 11 \mathrm{~b}^{+}$cells to IL-17 and TLR2 ligand stimulation in the presence of GM-CSF. Representative density-plot histograms showing apoptosis of neutrophils from A) PBS and B) ZIA group. Blood neutrophils incubated in medium containing GM-CSF $(50 \mathrm{ng} / \mathrm{ml})$ were stimulated with zymosan $(20 \mu \mathrm{g} / \mathrm{ml})$ in the absence or presence of IL-17 (40 ng/ml) and cultured at $37^{\circ} \mathrm{C}, 5 \% \mathrm{CO}_{2}$ for 24 hours. Apoptosis was measured by Annexin V-PI staining kit and analysed by flow cytometry. C) Effect of IL-17 and TLR2 on the frequencies of Annexin $\mathrm{V}^{+} \mathrm{Ly} 6 \mathrm{G}^{+}$cells in the GM-CSF loaded environment. Data represent the mean \pm SEM of Annexin $\mathrm{V}^{+}$cells ( $n=5$ animals per group in 3 experiments). $* p<0.05$, ***p $p<0.001$, Student's $t$ test.

pendant activation of cartilage chondrocytes and amplifying the IL-17 levels. Indeed neutrophils are a required source of IL-17 in the effector phase of arthritis [21]. Various studies have shown the ability of mouse neutrophils to produce IL-17 $[22,23]$. Human blood neutrophils are able to respond to 

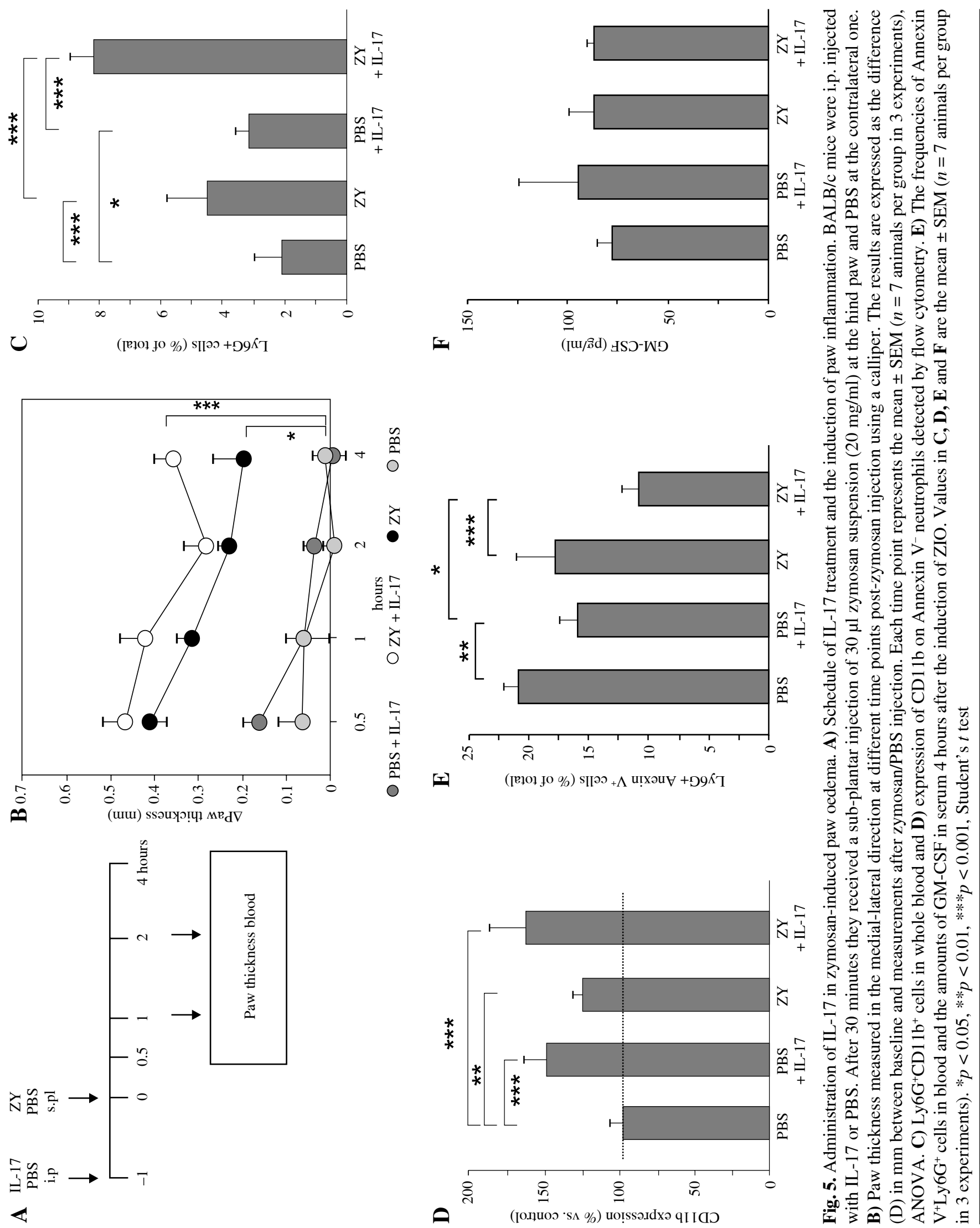
stimulation with expression of the transcripts encoding IL-17A and IL-17A protein as well [22]. Recently, more evidence has been found for the significance of IL-17 production by neutrophils in respect to certain disease pathologies.

Interleukin 17 stimulates granulopoiesis, neutrophil mobilisation from the bone marrow, enlarging the neutrophil circulatory pool. We hypothesised that IL-17 increases the numbers of Ly $6 \mathrm{G}^{+}$cells in blood not only by enhancing granulopoiesis but also by reducing or delaying the constitutive apoptosis. This hypothesis arises from studies showing a mechanism of IL-17-mediated survival in which chondrocytes undergo apoptosis when exposed to increasing concentrations of IL-17 [18]. Previously we observed that exogenous IL-17 induced IL-17 production in healthy neutrophils $[15,16]$. Nevertheless our present experiments showed that IL-17 was unable to change directly the dynamic of $\mathrm{Ly}_{6 \mathrm{G}}{ }^{+}$cell survival. Similarly, IL-17 fails to influence directly the constitutive apoptosis of human neutrophils [24]. It has been found that healthy neutrophils express the receptors for IL-17A and IL-17F [25] but not functional IL-17RC [24] that in turn may restrict the autocrine regulation of IL-17 production and IL-17-mediated apoptosis. A recent study showed that mouse $\mathrm{Ly} 6 \mathrm{G}^{+}$bone marrow cells up-regulate IL-17RA and IL-17RC genes, and human peripheral blood cells increased IL-17RC surface expression upon stimulation with IL-6 and IL-23, indicating that under certain conditions neutrophils can become sensitive to IL-17 [22]. We found that IL-17 in combination with zymosan reduced the apoptosis of neutrophils from controls as well as from arthritic mice in the 6-hour cultures. The synergistic action of both IL-17 and TLR2 ligand might be a result of various interactions at the protein level sharing common transduction pathways. The engagement of TLR2 on neutrophils delivers anti-apoptotic signals via NF- $\kappa B$ signalling pathway [26] and also enhances the expression of IL-17A receptor in a MyD88-dependent fashion [27]. Thus, neutrophils receive much stronger activation via both TLR2 ligand and IL-17 transduction pathways that can alter apoptosis as well. In fact, we found that IL-17 treatment of mice with zymosan-induced paw oedema enhanced the mobilisation of mature neutrophils into blood and their activation, and also decreased the frequencies of Annexin $\mathrm{V}^{+}$neutrophils.

In our study early apoptotic neutrophils showed less CD11b expression, unlike Annexin $\mathrm{V}^{-} \mathrm{Ly} 6 \mathrm{G}^{+}$cells. We think that this might be a mechanism to prevent the extravasation and trafficking of apoptotic neutrophils and to accelerate their elimination in blood. By contrast, Annexin $\mathrm{V}^{-}$neutrophils expressed CD11b highly. It has been shown that IL-17A stimulates CD11b expression in healthy human neutrophils [28]. In our in vitro cultures IL-17 alone elicited CD11b expression mainly on $\mathrm{Ly} 6 \mathrm{G}^{+}$cells from ZIA mice, demonstrating their increased sensitivity to the cytokines. However, the presence of both IL-17 and zymosan was able to enhance the level of surface CD11b on cells from the ZIA as well as from the PBS group.
CD11b expression was increased on blood $\mathrm{Ly}_{6 \mathrm{G}} \mathrm{G}^{+}$cells from IL-17-treated ZIO mice in which the frequencies of early apoptotic cells were reduced. Thus, we speculated that IL-17 can alter Ly6G ${ }^{+}$apoptosis by enhancing CD11b expression. Elicited density of CD11b on neutrophils can promote their adhesion to the endothelium [29]. Viable $\mathrm{CD}_{11} \mathrm{~b}^{+}$neutrophils, via activation of PI3K/Akt, MAPK/ ERK and NF- $\kappa \mathrm{B}$ survival pathways, retain their delayed apoptosis during the trans-endothelial migration [30, 31]. In respect to ZIA, infiltrating neutrophils exposed to IL-17 in the synovium can up-regulate CD11b allowing the interaction with myeloperoxidase. Such binding activates intrinsic anti-apoptotic pathways via caspases and Mcl-1, and postpones local neutrophil apoptosis [32].

The biological effects of IL-17 are often dependent on the production of GM-CSF that has anti-apoptotic action and/or increases cell survival [27]. In our 24-hour cell culture, where GM-CSF was present, IL-17 antagonised the action of TLR2 ligand. In the PBS group IL-17 increased the pool of TLR2-stimulated Annexin $\mathrm{V}^{+} \mathrm{Ly}_{6} \mathrm{G}^{+}$cells having a pro-apoptotic effect. By contrast, in the population from ZIA mice IL-17 reversed the action of zymosan, having an anti-apoptotic effect. We think these functional differences between PBS and ZIA neutrophils are due to their exposure to GM-CSF. Granulocyte-macrophage colony-stimulating factor production in serum differed between PBS- and zymosan-injected groups, and thus blood neutrophils from arthritic mice are likely primed by GM-CSF. In cultures GM-CSF can increase cell viability via multiple mechanisms interacting with IL-17R or TLR2 signalling. Recently it has been shown that IL-17A indirectly controls GM-CSF-mediated neutrophil survival, antagonising the various signal transduction pathways leading to the reduction of Mcl-1 protein level, aggregation of Bax to mitochondria and increased caspase-3/7 activity [25]. However, this interplay excludes death receptors cascade, suggesting that IL-17 antagonises mostly GM-CSF-dependant intrinsic apoptosis pathways. This might explain why we found reduced frequencies of Annexin ${ }^{+} \mathrm{Ly}_{6 \mathrm{G}}{ }^{+}$cells in IL-17-treated mice with ZIO despite similar serum GMCSF amounts in the experimental groups.

In conclusion, our study showed that IL-17 and TLR2-ligand (zymosan) can up-regulate CD11b expression and can modulate apoptosis of neutrophils from non-arthritic mice, arthritic mice and mice with paw oedema. This effect might be clinically relevant because of the increased neutrophil lifespan in circulation during chronic inflammatory conditions. It also suggests that anti-IL-17 therapy can be combined with inhibition of TLR2 and CD11b signalling in arthritis.

The authors declare no conflict of interest.

The work was financially supported by Inter-Pasteurien Concerted Actions Grant A05_11, France and by grant 
183/94/23551 from the Program BG051PO001/3.3-05-0001 of the Bulgarian Ministry of Education and Science and the European Social Fund.

\section{References}

1. Hotchkiss RS, Strasser A, McDunn JE, Swanson PE (2009): Mechanisms of disease: cell death. N Engl J Med 361: 15701583.

2. Akgul C, Moulding DA, Edwards SW (2001): Molecular control of neutrophil apoptosis. FEBS Lett 487: 318-322.

3. Croker BA, O'Donnell JA, Nowell CJ, et al. (2011): Fas-mediated neutrophil apoptosis is accelerated by Bid, Bak, and Bax and inhibited by Bcl-2 and Mcl-1. Proc Natl Acad Sci U S A 108: 13135-13140.

4. Raza K, Scheel-Toellner D, Lee CY, et al. (2006): Synovial fluid leukocyte apoptosis is inhibited in patients with very early rheumatoid arthritis. Arthritis Res Ther 8: R120.

5. Cascão R, Rosário HS, Souto-Carneiro MM, Fonseca JE (2010): Neutrophils in rheumatoid arthritis: More than simple final effectors. Autoimmun Rev 9: 531-535.

6. Cook AD, Pobjoy J, Sarros S, et al. (2013): Granulocyte-macrophage colony-stimulating factor is a key mediator in inflammatory and arthritic pain. Ann Rheum Dis 72: 265-270.

7. Kobayashi SD, Voyich JM, Whitney AR, DeLeo FR (2005): Spontaneous neutrophil apoptosis and regulation of cell survival by granulocyte macrophage-colony stimulating factor. J Leuk Biol 78: 1408-1418.

8. Moulding DA, Akgul C, Derouet M, et al. (2001): BCL-2 family expression in human neutrophils during delayed and accelerated apoptosis. J Leuk Biol 70: 783-792.

9. Klein JB, Rane MJ, Scherzer JA, et al. (2000): Granulocyte-macrophage colony-stimulating factor delays neutrophil constitutive apoptosis through phosphoinositide 3-kinase and extracellular signal-regulated kinase pathways. J Immunol 164: 4286-4291.

10. Kotone-Miyahara Y, Yamashita K, Lee KK, et al. (2004): Short-term delay of Fas-stimulated apoptosis by GM-CSF as a result of temporary suppression of FADD recruitment in neutrophils: evidence implicating phosphatidylinositol 3-kinase and MEK1-ERK1/2 pathways downstream of classical protein kinase C. J Leuk Biol 76: 1047-1056.

11. van den Berg WB, Miossec P (2009): IL-17 as a future therapeutic target for rheumatoid arthritis. Nat Rev Rheumatol 5: 549-553.

12. Hu Y, Shen F, Crellin NK, Ouyang W (2011): The IL-17 pathway as a major therapeutic target in autoimmune diseases. Ann N Y Acad Sci 1217: 60-76.

13. Chabaud M, Lubberts E, Joosten L, et al. (2001): IL-17 derived from juxta-articular bone and synovium contributes to joint degradation in rheumatoid arthritis. Arthritis Res 3: 168-177.

14. Kokubu T, Haudenschild DR, Moseley TA, et al. (2008): Immunolocalization of IL-17A, IL-17B, and their receptors in chondrocytes during fracture healing. J Histochem Cytochem 56: 89-95.

15. Milanova V, Ivanovska N, Dimitrova P (2012): Neutrophils are the source of IL-17 and RANKL in zymosan induced arthritis. Arthritis Res Ther 14: P46.

16. Milanova V, Ivanovska N, Dimitrova P (2014): TLR2 elicits IL-17-mediated RANKL expression, IL-17, and OPG production in neutrophils from arthritic mice. Mediators Inflamm 2014: 14 .
17. Luo Y, Dorf ME. Isolation of mouse neutrophils. Current protocols in immunology. John Wiley \& Sons, Inc., New York 2001 .

18. Koenders MI, Kolls JK, Oppers-Walgreen B (2005): Interleukin-17 receptor deficiency results in impaired synovial expression of interleukin-1 and matrix metalloproteinases 3, 9, and 13 and prevents cartilage destruction during chronic reactivated streptococcal cell wall-induced arthritis. Arthritis Rheum 52: 3239-3247.

19. Kokkonen H, Söderström I, Rocklöv J, et al. (2010): Up-regulation of cytokines and chemokines predates the onset of rheumatoid arthritis. Arthritis Rheum 62: 383-391.

20. Moran EM, Heydrich R, Ng CT, et al. (2011): IL-17A expression is localised to both mononuclear and polymorphonuclear synovial cell infiltrates. PLoS One 6: e24048.

21. Katayama M, Ohmura K, Yukawa N, et al. (2013): Neutrophils are essential as a source of IL-17 in the effector phase of arthritis. PLoS One 8: e62231.

22. Taylor PR, Roy S, Leal SM Jr (2014): Activation of neutrophils by autocrine IL-17A-IL-17RC interactions during fungal infection is regulated by IL-6, IL-23, RORgammat and dectin-2. Nat Immunol 15: 143-151.

23. Keijsers RR, Hendriks AG, van Erp PE, et al. (2013): In vivo induction of cutaneous inflammation results in the accumulation of extracellular trap-forming neutrophils expressing RORgammat and IL-17. J Invest Dermatol 6: 526.

24. Pelletier M, Maggi L, Micheletti A, et al. (2010): Evidence for a cross-talk between human neutrophils and Th17 cells. Blood 115: 335-343.

25. Dragon S, Saffar AS, Shan L, Gounni AS (2008): IL-17 attenuates the anti-apoptotic effects of GM-CSF in human neutrophils. Mol Immunol 45: 160-168.

26. Lotz S, Aga E, Wilde I, et al. (2004): Highly purified lipoteichoic acid activates neutrophil granulocytes and delays their spontaneous apoptosis via CD14 and TLR2. J Leukoc Biol 75: 467-477.

27. Da Silva CA, Hartl D, Liu W, et al. (2008): TLR-2 and IL$17 \mathrm{~A}$ in chitin-induced macrophage activation and acute inflammation. J Immunol 181: 4279-4286.

28. Taooka Y, Ohe M, Chen L, et al. (2013): Increased expression levels of integrin alpha9beta1 and CD11b on circulating neutrophils and elevated serum IL-17A in elderly aspiration pneumonia. Respiration 86: 367-375.

29. Luo BH, Carman CV, Springer TA (2007): Structural basis of integrin regulation and signaling. Annu Rev Immunol 25: 619-647.

30. Whitlock BB, Gardai S, Fadok V, et al. (2000): Differential roles for alpha(M)beta(2) integrin clustering or activation in the control of apoptosis via regulation of akt and ERK survival mechanisms. J Cell Biol 151: 1305-1320.

31. Rubel C, Gómez S, Fernández GC, et al. (2003): Fibrinogen-CD11b/CD18 interaction activates the NF-kappa B pathway and delays apoptosis in human neutrophils. Eur J Immunol 33: 1429-1438.

32. El Kebir D, József L, Pan W, Filep JG (2008): Myeloperoxidase delays neutrophil apoptosis through CD11b/CD18 integrins and prolongs inflammation. Circ Res 103: 352-359. 